\section{Le point faible méiotique : la première division}

\author{
M. Emilie Terret, Katja Wassmann
}

> La méiose est une succession de deux divisions sans synthèse d'ADN intermédiaire, conduisant à la formation de gamètes haploïdes fécondables et fécondants : les ovocytes et les spermatozoïdes. La première division permet la séparation des chromosomes homologues, la seconde celle des chromatides sœurs. La première division méiotique femelle est sujette à des erreurs de ségrégation du matériel génétique, qui ont de lourdes conséquences pour l'embryon (génération d'embryons aneuploïdes, avortements spontanés, trisomies). Nous décrivons dans cet article les avancées récentes concernant la régulation de cette première division, en particulier celles liées à l'assemblage des fuseaux de division et à la régulation du cycle cellulaire méiotique chez la souris. <

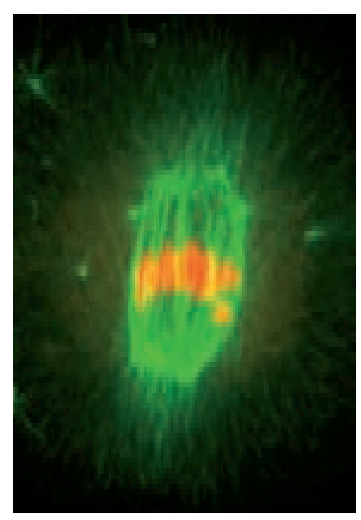

M.E. Terret: Molecular Biology Program, Memorial

Sloan-Kettering Cancer Center, Box 97, 1275 York Avenue, 10021 New York, Ny, États-Unis.

K. Wassmann : Team Avenir Inserm, Cell Division and Associated Checkpoints. UMR 7622 CNRS, Biologie du Développement, Université Pierre et Marie Curie, 9, quai Saint-Bernard, Boîte 24, 75005 Paris, France.

katja.wassmann@snv.jussieu.fr

La maturation méiotique:

une succession de deux divisions sans synthèse d'ADN intermédiaire

La maturation méiotique marque l'achèvement de la méiose depuis la levée du blocage en prophase de première division de méiose jusqu'à la fécondation. L'ovocyte de souris est le modèle étudié pour ces questions le plus proche de l'homme, et les ovocytes sont transparents, ce qui rend l'architecture intérieure de la cellule facilement observable. Enfin c'est un modèle où des approches génétiques sont possibles. La maturation méiotique est schématisée dans la Figure l. L'ovocyte immature de souris est bloqué en prophase I de méiose dans les ovaires; il est caractérisé morphologiquement par la présence d'un noyau, la vésicule germinative (GV, germinal vesicle). Cet arrêt est levé par une stimulation hormonale qui déclenchera indirectement la reprise de la méiose. Le premier évènement morphologique visible marquant l'entrée en maturation méiotique est la rupture de l'enveloppe nucléaire (GVBD, germinal vesicle breakdown). La chromatine se condense, le réseau de microtubules se réorganise et un fuseau de métaphase I se forme en position centrale. Ce fuseau migre vers le cortex de l'ovocyte, permettant, après séparation des chromosomes homologues, une première division asymétrique. Cette division donne lieu à une petite cellule -le globule polaire- et une grosse cellule -l'ovocyte. L'asymétrie de cette division permet l'accumulation de 
la majorité des réserves nécessaires au développement futur de l'embryon dans l'ovocyte. L'ovocyte entre en seconde division de méiose sans passer par une phase $\mathrm{S}$ intermédiaire, étape fondamentale à l'obtention d'un gamète haploïde. II forme un fuseau de métaphase II en position sous-corticale et reste arrêté en métaphase II jusqu'à la fécondation grâce à une activité appelée CSF (cytostatic factor). Cet arrêt en métaphase de seconde division méiotique est caractéristique des ovocytes de vertébrés. II est caractérisé par une forte activité MPF (M-phase promoting factor) et par la présence d'un fuseau de division stable avec des chromosomes alignés sur la plaque équatoriale. Le MPF est le facteur universel d'entrée en phase $M$ de toutes les cellules eucaryotes, il est constitué de deux sous-unités, la Cycline B et Cdc2. L'activité CSF ralentit la dégradation de la Cycline $B$, maintenant ainsi une activité MPF forte qui empêche la sortie de métaphase. La fécondation déclenche la sortie de cette phase d'arrêt et entraîne l'achèvement de la méiose avec la séparation des chromatides sœurs et l'expulsion du second globule polaire (pour revue voir $[4,5]$ ).

\section{Régulation de la sortie de méiose I}

Chez la femme, le risque d'erreurs lors de la méiose augmente avec l'âge. Le plus souvent il s'agit d'erreurs de ségrégation des chromosomes en méiose I, induisant l'aneuploïdie des ovocytes, et en conséquence celle des embryons. Une bonne revue a été publiée sur l'âge maternel et les anomalies chromosomiques dans les ovocytes humains [6]. Quand l'âge augmente, la ségrégation des chromosomes devient de moins en moins fidèle,

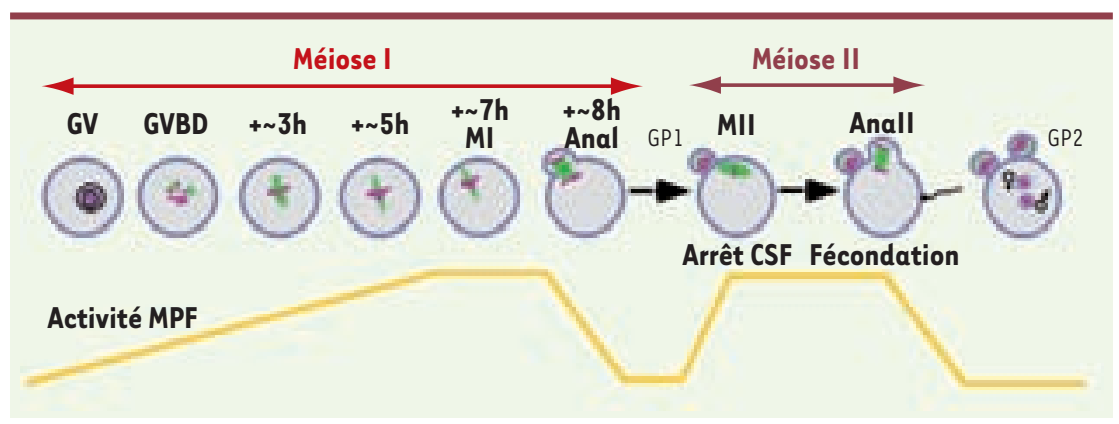

Figure 1. La maturation méiotique chez la souris. L'ovocyte est bloqué en prophase de première division de méiose dans les ovaires au stade vésicule germinative (GV : germinal vesicle). En réponse à une stimulation hormonale, la méiose reprend, induisant la rupture de la vésicule germinative (GVBD : germinal vesicle breakdown). L'ADN s'organise en chromosomes condensés qui s'alignent sur le fuseau de division au cours de la métaphase de première division méiotique (MI). Le fuseau migre au cortex, les chromosomes homologues sont ségrégés, et le premier globule polaire est expulsé (GPI) au cours de l'anaphase I (Anal). L'ovocyte entre en seconde division méiotique sans réplication d'ADN intermédiaire ; le fuseau se reforme en position souscorticale, et les chromatides sœurs s'alignent sur ce fuseau au cours de la métaphase (MII). L'ovocyte reste bloqué en MII grâce à l'activité CSF (cytostatic factor) jusqu'à la fécondation, qui va déclencher l'achèvement de la méiose avec la séparation des chromatides sœurs, et la formation et l'expulsion du second globule polaire (GP2) à l'anaphase II (Anall). L'ADN décondense, et les deux pronucléus (mâle et femelle) qui vont fusionner sont visibles dans l'ovocyte. Les microtubules sont en vert, et l'ADN en violet. La courbe représente le niveau d'activité MPF (M-phase promoting factor, en jaune). ce qui peut être une conséquence de défauts de cohésion des chromatides sœurs [7], de défauts de recombinaison méiotique [3], mais peut-être aussi de défauts de la machinerie de ségrégation comme le fuseau de division, ou des mécanismes de régulation de la ségrégation. Des avancées récentes dans ces deux domaines ont permis de mieux appréhender la formation des fuseaux de division mieux comprendre et éventuellement traiter les aneuploï-

\section{Le fuseau de méiose I :}

un outil essentiel pour assurer une division équivalente du matériel génétique

Les fuseaux mitotiques et méiotiques sont des structures bipolaires très dynamiques formées de microtubules arrangés de façon symétrique et fusiforme. Au cours de la division, les chromosomes s'alignent progressivement sur des fuseaux en mitose. La première implique les centrosomes. Les centrosomes sont composés d'une paire de centrioles (eux-mêmes formés de cylindres de microtubules) entourés de matériel péri-centriolaire capable de nucléer les microtubules. Au cours de la division mitotique, la cellule possède deux centrosomes. Ces centrosomes nuclééent des microtubules astraux qui cherchent et capturent les chromosomes, définissant ainsi les deux pôles du fuseau mitotique et permettant l'alignement des chromosomes sur la plaque équatoriale du fuseau [8]. La seconde voie est dépendante du gradient de la GTPase Ran sous sa forme active couplée au GTP (pour revue voir [9]). La production de Ran-GTP dépend de l'activité de son facteur d'échange RCCl. $\mathrm{RCCl}$ est lié à la chromatine en mitose, ce qui permet la formation d'un gradient de Ran-GTP autour des chromosomes (la plus forte concentration de Ran-GTP étant au niveau des chomomomes, et la moins forte dans le cytoplasme). Ran-GTP libère les SAF (spindle assembly factors) - des facteurs qui stimulent l'assemblage des microtubules - de l'effet inhibiteur des importines, et favorise donc ainsi la formation des microtubules et plus largement du fuseau à proximité des chromosomes (Figure 2). 
Des études effectuées sur des ovocytes humains ont montré que les fuseaux de division méiotiques sont souvent aberrants chez les femmes âgées, pouvant ainsi contribuer aux aneuploïdies ovocytaires liées à l'âge chez la femme [10]. La formation des fuseaux de division méiotique a été décrite précisément chez la souris, mais les mécanismes moléculaires sont restés longtemps élusifs (pour revue voir [4]). Des analyses récentes en microscopie confocale quantitative haute résolution réalisées dans des ovocytes de souris ont montré que la nucléation des microtubules en méiose I se fait à partir de l'auto-assemblage de 80 centres organisateurs des microtubules, qui en quelque sorte remplacent les centrosomes en formant un réseau cytoplasmique de microtubules en prophase, permettant la formation d'un fuseau bipolaire [11]. Le fuseau est mis en place deux heures après la rupture de l'enveloppe nucléaire et reste en place tout au long de la très longue prométaphase (qui dure six heures chez la souris), jusqu'à l'anaphase qui a lieu six à sept heures plus tard [12]. Le fuseau de la seconde division de méiose se forme rapidement à partir des microtubules qui sont restés à proximité des chromosomes après la première division de méiose, et il est stable au cours de l'arrêt CSF [13]. Les fuseaux méiotiques sont dépourvus de centrioles, ce qui exclut donc la voie centrosomique active en mitose comme mécanisme permettant de former les fuseaux en méiose. La voie dépendante du gradient de la GTPase Ran paraît donc une bonne candidate pour expliquer la formation des fuseaux en méiose. Chez les vertébrés, cette voie a été largement étudiée in vitro dans des extraits d'ovocytes de xénope, mais son rôle n'avait pas été étudié in vivo en méiose. Récemment, il a été

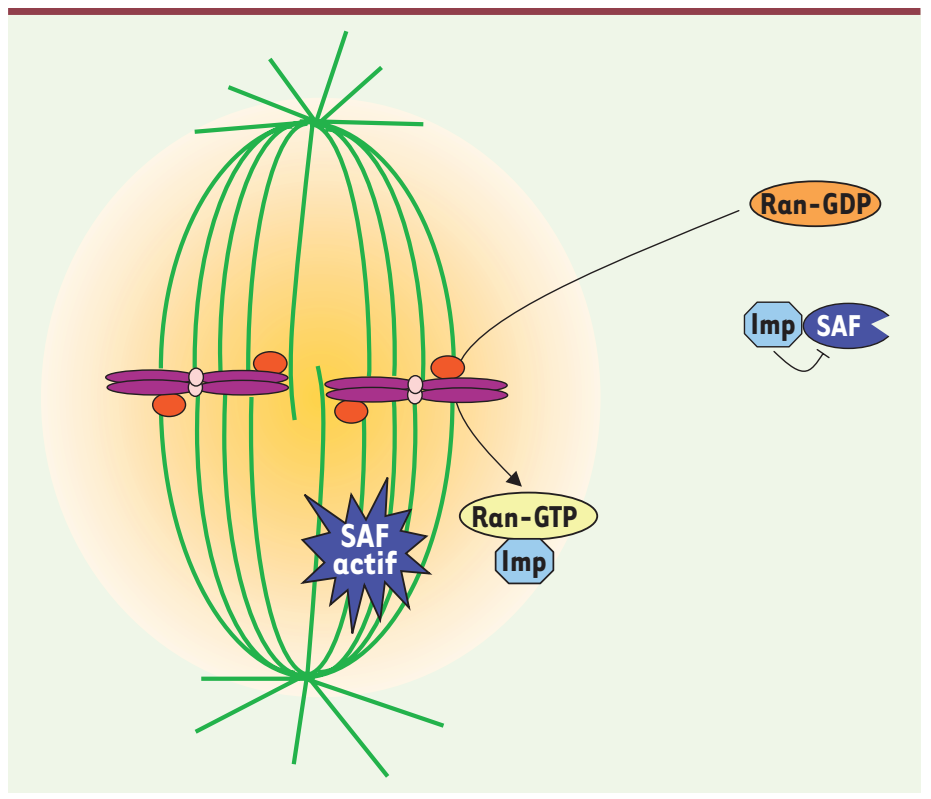

Figure 2. Formation du fuseau mitotique par la voie Ran. RCCl (en rouge) est le facteur d'échange de Ran-GTP. Il est lié aux chromosomes en mitose, induisant la formation d'un gradient de Ran-GTP autour des chromosomes (halo jaune). Ran sous sa forme GTP induit la formation de microtubules à proximité des chromosomes, en libérant de l'effet inhibiteur des Importines (en bleu pâle) des facteurs d'assemblage des microtubules (SAF, spindle assembly factors, en bleu foncé). Loin des chromosomes, Ran est sous forme GDP et ne peut donc pas libérer ces facteurs des Importines, inhibant donc la formation des microtubules. montré qu'un gradient de Ran-GTP est établi en méiose I, et qu'il perdure autour des chromosomes jusqu'en méiose II. Chez le xénope et la souris, la formation du fuseau de méiose I semble insensible à des modifications du gradient de Ran-GTP, et ce malgré l'absence de centrioles, donc en l'absence de la voie centrosomale. II existe par conséquent un mécanisme autre que les deux voies classiques, capable de contribuer à la formation d'un fuseau de division en méiose I $[11,13]$. La formation du fuseau de méiose II est sensible au gradient de Ran-GTP, et le fuseau a besoin d'être stabilisé dans le temps, via les protéines MISS (MAPK-interacting and spindle-stabilizing protein) et DOCIR (Deleted in oral cancer one related) [14, 15]. Une fois les fuseaux de division formés, les chromosomes s'alignent sur la plaque métaphasique, jusqu’à leur séparation à l'anaphase.

\section{Le matériel génétique ségrégé en méiose I : les chromosomes homologues}

Une différence essentielle entre la maturation méiotique et la mitose est que lors de la méiose I, les chromosomes homologues paternels et maternels sont ségrégés entre l'ovocyte et le globule polaire, alors qu'en mitose, les chromatides sœurs formant un chromosome sont séparées entre les deux cellules filles (Figure 3) [16]. En mitose, les deux chromatides sœurs composant un chromosome sont attachées au fuseau bipolaire par leurs kinétochores. Les kinétochores d'une paire de chromatides sœurs sont orientés vers les pôles opposés du fuseau bipolaire, permettant ainsi de générer la tension nécessaire à la séparation des chromatides sœurs entre les deux cellules filles à la transition métaphase-anaphase (Figure 3). Jusqu'à cette transition, les chromatides sœurs sont maintenues ensemble grâce aux cohésines, qui formeraient un anneau autour d'elles, leur permettant de rester appariées jusqu'à leur séparation à l'anaphase [17]. En méiose I, ce sont les chromosomes homologues qui sont appariés, et les deux kinétochores d'un chromosome (formé de deux chromatides sœurs) sont orientés vers le même pôle, contrairement à la mitose (Figure 3). En prophase de méiose, avant la maturation méiotique, les chromosomes homologues échangent du matériel génétique. Ce processus est appelé recombinaison méiotique et créé des liens physiques entre les chromosomes, au niveau des chiasmas. La séparation des chromosomes en méiose I requiert la disparition des cohésines reliant les deux chromosomes à l'endroit où la recombinaison a eu lieu, et plus largement sur les bras des chromatides sœurs. Les cohésines entourant les centromères de deux chromatides sœurs doivent être protégées afin de leur permettre de rester appariées jusqu'à la méiose II (Figure 3) [16]. Les protéines appelées Shugoshin sont responsables de cette protection [18]. La méiose II ressemble 
à une division mitotique, car ce sont des chromatides sœurs qui sont appariées et séparées à l'anaphase, comme en mitose. Les mécanismes contrôlant le cycle cellulaire mitotique sont-ils à l'œuvre en méiose I, division méiotique la plus éloignée d'une mitose en terme chromosomique?

\section{L'APC/C : le chef d'orchestre mitotique}

Pour comprendre comment est contrôlé le cycle cellulaire méiotique, il est impératif d'introduire les mécanismes contrôlant le cycle cellulaire mitotique. Des étapes essentielles de la mitose sont contrôlées par un complexe multiprotéique, la $\varepsilon 3$ ubiquitine ligase ou APC/C (anaphase promoting complex/cyclosome), qui est requis pour ubiquitinyler certaines protéines dont la dégradation est nécessaire au déclenchement de l'anaphase. L'ubiquitinylation par l'APC/C dirige en effet les protéines ainsi étiquetées vers le protéasome $26 \mathrm{~S}$ qui les dégrade. L'APC/C a la capacité d'ubiquitinyler des substrats variés à différentes phases de la mitose. Sa spécificité dépend de son association avec des activateurs et inhibiteurs spécialisés (Figure 4A). En pro-métaphase de la division mitotique, la Cycline A est ubiquitinylée et dégradée grâce à l'APC/C associé à l'activateur Cdc20, induisant la disparition de l'activité kinasique associée à la Cycline A. À ce stade, l'APC/C est sensible à l'inhibiteur Emil. La progression de la division mitotique est accompagnée par l'accumulation de l'activité MPF. Une chute de l'activité MPF est observée juste avant l'anaphase. Cette chute est due à l'ubiquitinylation de la Cycline B, composante du MPF, par l'APC/C associé à l'activateur Cdc20, et elle est sensible à l'inhibiteur Mad2. Avant d'entrer en phase Gl, toute activité kinasique associée au MPF doit disparaître. La cellule accomplit cette tâche via un deuxième activateur de l'APC/C, Cdhl. Cdhl est inhibé par phosphorylation par le MPF; la première vague de dégradation de la Cycline B suffit pour permettre l'activation de Cdhl, qui va ensuite cibler les molécules de Cycline B restantes. Un autre substrat clef de la transition métaphase-anaphase est la Sécurine, un inhibiteur d'une protéase, la Séparase. Après la dégradation de la Sécurine

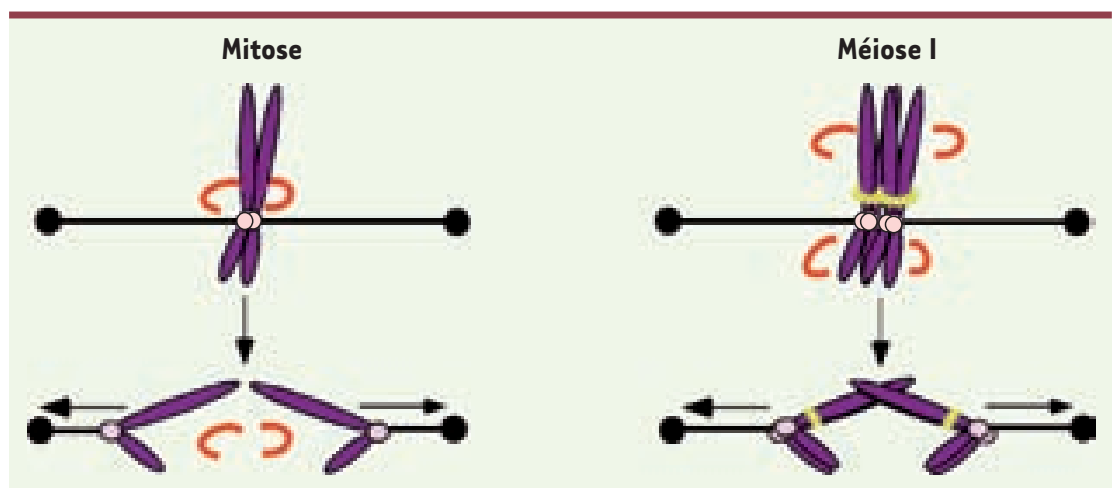

Figure 3. Transition métaphase/anaphase en mitose et méiose I. En mitose, les chromatides sœurs formant un chromosome sont séparées entre les deux cellules filles. Lors de la première division méiotique, les chromosomes homologues paternels et maternels sont ségrégés entre l'ovocyte et le globule polaire. Les cohésines qui doivent être clivées pour permettre la séparation des chromatides sœurs en mitose et des chromosomes homologues en méiose I sont en rouge. Les cohésines qui doivent rester associées avec les chromatides sœurs composant un chromosome, leur permettant de rester liées jusqu'en méiose II, sont en vert. L'ADN est en violet et les kinétochores en rose. en métaphase, la Séparase est libérée et peut cliver sa cible, la sous-unité Sccl du complexe de cohésines. Une fois Sccl clivée, l'anneau de cohésines qui maintient les chromatides sœurs ensemble est détruit [19].

Au cours de la division mitotique, un mécanisme de contrôle appelé point de contrôle du fuseau empêche le déclenchement de l'anaphase tant que tous les chromosomes ne sont pas attachés au fuseau mitotique et alignés sur la plaque équatoriale sous tension [20]. Cela permet d'éviter une séparation précoce et anarchique des chromatides sœurs, et d'assurer que le contenu en ADN est bien séparé en deux fractions égales à l'issue de la division cellulaire. Durant la phase d'attachement et d'alignement des chromosomes, I'APC/C est inhibé par le point de contrôle du fuseau via Mad2. Le MPF reste donc actif et les chromatides sœurs restent liées entre elles. L'alignement du dernier chromosome sur la plaque métaphasique du fuseau va inactiver le point de contrôle du fuseau et ainsi activer l'APC/C. L'APC/C actif ubiquitinyle ses substrats (la Sécurine et la Cycline B), les ciblant pour dégradation par le protéasome $26 \mathrm{~S}$, et induit donc la séparation des chromatides sœurs, la chute de l'activité MPF, et la sortie de phase M (Figure 4B). Le point de contrôle du fuseau coordonne donc la phase de capture des chromosomes, l'activité du MPF et la séparation physique des chromatides sœurs.

\section{En méiose, \\ un chef d'orchestre controversé}

En méiose I, des résultats contradictoires selon les espèces ont été publiés quant à l'existence d'un mécanisme de contrôle de type mitotique passant par l'inhibition de I'APC/C et contrôlant la séparation des chromosomes homologues. Chez des espèces comme la levure et le nématode, il a été montré que I'APC/C actif et la Séparase sont requis pour effectuer la première division de méiose, alors que chez le xénope, I'APC/C actif n'est pas requis (pour revue voir [21]). Chez la souris, la fonctionnalité d'un point de contrôle du fuseau était très controversée, notamment par le fait qu'il existe des souris $X O$ ayant un seul chromosome $X$ et cependant parfaitement fertiles [22] (au cours de la division mitotique, la cellule serait bloquée en métaphase en réponse à l'activation du point de contrôle du fuseau dans une situation similaire). Enfin il existe une forte corrélation entre l'âge des femmes et le pourcentage d'aberrations 


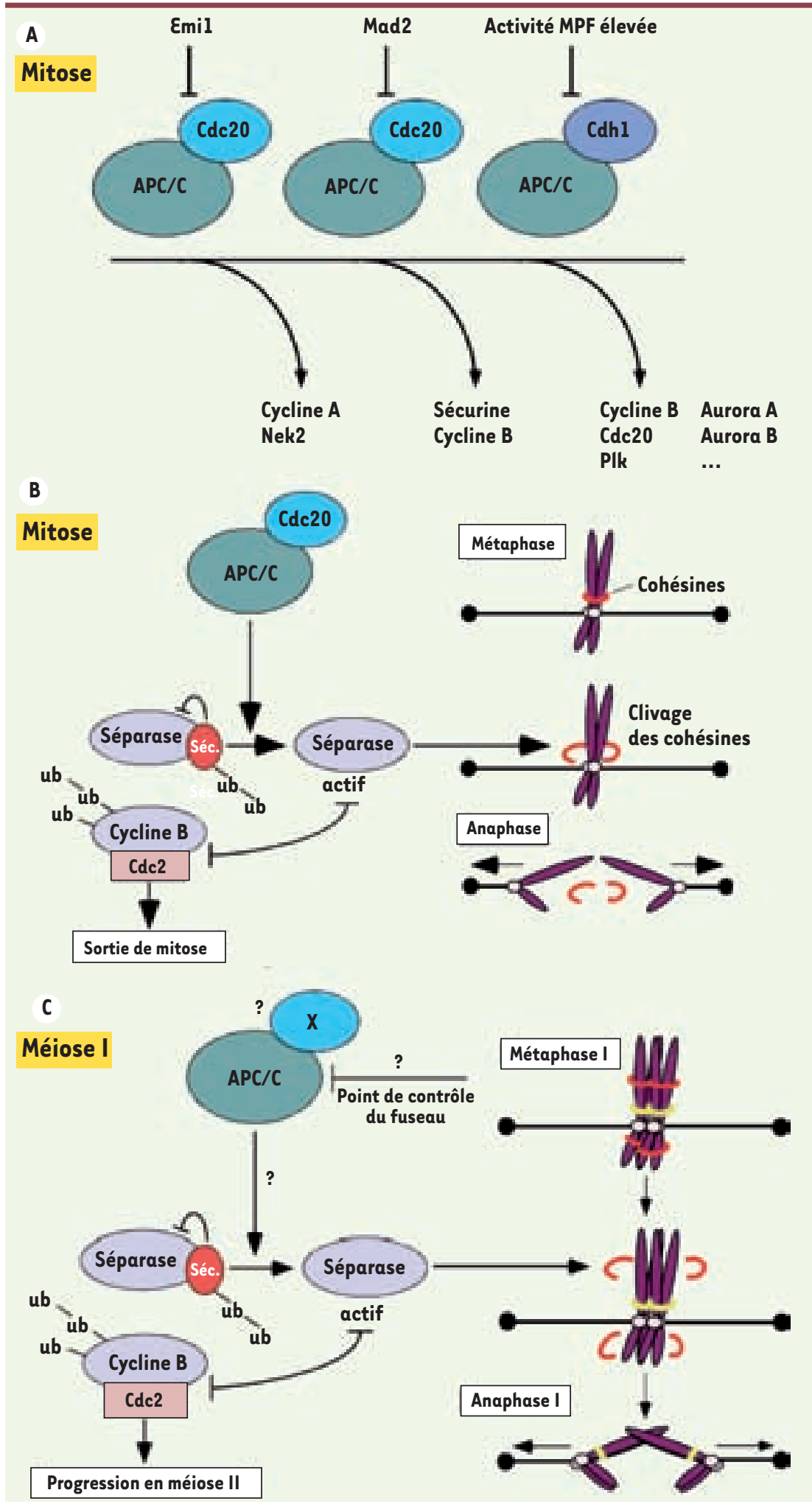

chromosomiques résultant d'erreurs au cours de la méiose I femelle, suggérant qu'aucun mécanisme de contrôle n'arrête la division méiotique chez l'homme en cas d'erreurs comme c'est le cas au cours de la division mitotique, ou bien que son efficacité diminue en fonction de l'âge [3]. Ces erreurs induisent l'aneuploïdie des ovocytes, et donc celle des embryons.

Un ensemble d'études récentes suggère que la dégradation induite par I'APC/C et un mécanisme de contrôle de type mitotique existent et seraient fonctionnels en méiose I chez la souris (Figure 4C). Tout d'abord la dégradation induite par le protéasome est requise pour la sortie de méiose I, comme I'a montré l'utilisation d'un inhibiteur du protéasome qui bloque les ovocytes en méiose I [23]. Plus précisément, la Cycline B

Figure 4. L'APC/C, le point de contrôle du fuseau et la transition métaphase/ anaphase en mitose et méiose I. A. La régulation de l'APC/C en mitose. L'APC/C a la capacité d'ubiquitinyler des substrats variés à différentes phases de la mitose. Sa spécificité dépend de son association avec des activateurs ( $\mathrm{Cdc} 20, C \mathrm{dhl})$ et inhibiteurs ( $\varepsilon$ mil, Mad2) spécialisés. En début de mitose, l'APC/CCdc20 cible la Cycline A et Nek2; I'APC/C est alors sensible à Emil. À la transition métaphase/ anaphase, I'APC/C-Cdc20 cible la Sécurine et la Cycline B. I'APC/C est alors sensible à Mad2. A la sortie de mitose, l'APC/C-Cdhl cible la Cycline B, Cdc20, Aurora A, Aurora B, Polo kinase (PIk) ... $B$. La régulation de la transition métaphase/ anaphase en mitose. Le point de contrôle du fuseau surveille que tous les chromosomes sont alignés et sous tension sur la plaque métaphasique du fuseau. Quand c'est le cas, l'APC/C est actif et ubiquitinyle la Sécurine et la Cycline B. La Séparase devient active et clive les cohésines, permettant la ségrégation des chromatides sœurs entre les deux cellules filles. L'activité MPF chute, entraînant la sortie de mitose. C. La régulation de la transition métaphase/anaphase en méiose I. Comme en mitose, la dégradation de la Sécurine et de la Cycline B est requise pour la transition métaphase/anaphase, afin de permettre l'activation de la Séparase et la progression en méiose II. II a été montré que la Séparase est nécessaire pour cliver les cohésines sur les bras de chromosomes. Cependant, il n’y a pas de preuve directe montrant que c'est l'APC/C qui est responsable de la dégradation de la Sécurine et de la Cycline B. De même, il n'a pas été montré directement que le point de contrôle du fuseau cible l'APC/C en méiose I. De plus il reste à déterminer s'il existe un activateur de l'APC/C spécifique de la méiose I dans les ovocytes de mammifères. 
et la Sécurine doivent être dégradées à la transition métaphase-anaphase, leur surexpression bloquant également les ovocytes de souris en méiose I [23-25]. Ensuite, la dérégulation de modulateurs de l'APC/C compromet la sortie de méiose I. La surexpression de Mad2 entraine un arrêt en méiose I, et l'expression d'une forme mutée dominante négative de Mad2 (mimant la forme phosphorylée de la protéine) entraîne la sortie de méiose I avec des défauts de ségrégation des chromosomes [26]. Le même résultat est obtenu après expression de siARN ciblant Mad2 : la Cycline B et la Sécurine sont dégradées précocement, et les ovocytes sortent de méiose I trop tôt [27]. De même l'expression de mutants dominants négatifs de protéines du point de contrôle du fuseau comme Bubl et BubRl, de mutants de Cdc20 actifs, ou la perte d'un allèle de Mad2 dans les ovocytes issus de souris invalidées pour Mad2 de manière hétérozygote [28] accélèrent la transition métaphase-anaphase en méiose I; en conséquence, les ovocytes ségrègent mal leurs chromosomes homologues [29]. Enfin, un des effecteurs du point de contrôle du fuseau, la Séparase, doit être correctement régulé pour sortir de méiose I. Un inhibiteur peptidique spécifique de la Séparase exprimé en méiose I induit des erreurs de ségrégation, visibles en méiose II par la présence de chromosomes homologues non disjoints [23]. La résolution de l'anneau de cohésines autour des chromosomes en méiose I semble donc dépendre de la Séparase. L'analyse de souris porteuses d'une construction permettant l'invalidation conditionnelle (knock-out conditionnel) de la Séparase sélectivement dans les ovocytes l'a montré de manière indiscutable [30]. Les cohésines présentes en méiose ont une sous-unité méiotique spécifique, Rec8, et c'est cette sous-unité qui est clivée le long des bras des chromosomes, mais pas autour des centromères en méiose I. Les ovocytes issus des souris invalidées pour la Séparase ne peuvent pas cliver Rec 8 en méiose I, et n'expulsent pas de premier globule polaire; ces souris sont stériles [30]. Contrairement à ce qui se passe au cours de la mitose, la Séparase semble aussi avoir un rôle au cours de la cytokinèse en méiose I, car, indépendamment de son activité protéasique, elle peut promouvoir l'expulsion d'un globule polaire sans séparation des chromosomes homologues $[30,31]$.

En résumé, l'inhibition de la dégradation induite par le protéasome, l'inhibition concomitante mais mutuellement indépendante de la dégradation de la Sécurine et de la Cycline $B$ en particulier, la dérégulation de modulateurs de l'APC/C, et la dérégulation de l'activité de la Séparase, altèrent la transition méiose I/méiose II. Cela suggère que l'APC/C et le point de contrôle du fuseau sont fonctionnels et requis chez la souris pour orchestrer la transition méiose I/méiose II. Ce mécanisme de contrôle semble donc conservé entre les espèces, et entre la mitose et la méiose, à l'exception du xénope.

\section{Questions ouvertes et paradoxes méiotiques}

La régulation directe de l'APC/C en méiose I dans les ovocytes de mammifères reste cependant obscure. Les preuves montrant que l'APC/C est requis en méiose I sont indirectes, et aucun activateur de l'APC/C spécifique de la méiose n'a été mis en évidence chez les mammifères, comme cela a été le cas dans d'autres espèces [32]. II semblerait que l'activateur requis pour l'entrée en méiose I soit Cdhl [33]. Un rôle difficile à concilier avec le fait que $\mathrm{Cdhl}$ est requis pour la sortie de mitose, et que des substrats de l'APC/ $C$ qui sont dégradés de manière dépendante de $C d h l$ peuvent cependant s'accumuler à l'entrée en méiose I, comme par exemple Cdc20 et la kinase Polo. Une autre question intéressante concerne Mad2. Comme en mitose, Mad2 semble être impliquée dans la détection des attachements incorrects entre microtubules et kinétochores en méiose I. II reste à déterminer comment Mad2 est capable de reconnaître un attachement correct en méiosel, sachant que le même type d'attachement (c'est-à-dire les deux kinétochores de deux chromatides sœurs attachés au même pôle du fuseau de division) est reconnu comme erroné en mitose par la même protéine. La formation atypique du fuseau de méiose I pourrait-elle lui conférer des propriétés intrinsèques lui permettant de reconnaître l'attachement des chromosomes homologues au même pôle (chromatides sœurs avec la même orientation) comme correct? Quel serait alors le rôle de Ran en méiose I ? Chez la souris, il a été montré récemment que Ran pourrait jouer un rôle dans l'établissement de l'asymétrie de la première division méiotique [34]. Dans le futur, des études combinant la génétique et la biologie cellulaire permettront d'élucider ces questions importantes. $\diamond$

\section{Prise de distance}

Chez la femme, entre 35 et 45 ans, le risque d'accidents en méiose augmente dramatiquement, la fréquence de trisomies passant de 7 à $35 \%$. Le plus souvent il s'agit d'erreurs de ségrégation des chromosomes au cours de la méiose I, induisant l'aneuploïdie des ovocytes et donc des embryons, conduisant à des problèmes de stérilité féminine ainsi qu'à la formation d'embryons trisomiques. Cet effet de l'âge maternel sur la survenue des non-disjonctions chromosomiques est un phénomène multifactoriel, faisant intervenir des facteurs intrinsèques aux mécanismes moléculaires contrôlant la méiose, mais aussi des facteurs environnementaux. Par conséquent, il est essentiel de comprendre comment les divisions méiotiques sont contrôlées. Les études moléculaires menées sur les ovocytes humains sont fondamentales à la compréhension de ces mécanismes, mais il y a des biais expérimentaux incontournables. La plupart du temps les ovocytes analysés sont issus d'échecs de fécondation in vitro (FIV), et ont donc été obtenus après hyperstimulation ovarienne et manipulations in vitro, ce qui peut fausser les résultats de leur analyse. Il est également difficile de faire des études statistiquement significatives, étant donné la difficulté d'obtention des ovocytes humains. Des approches scientifiques récentes chez la souris en particulier ont permis de progresser dans ce domaine encore relativement mal compris. Dans le futur la compréhension des mécanismes assurant la formation d'ovocytes matures fécondables chez la souris aidera sans doute à appréhender chez l'homme les problèmes de stérilité féminine, et de génération de trisomies. 


\section{SUMMARY}

Meiotic weakness : the first division

Cell division is probably the most dramatic event in the life of a cell : the entire genetic material has to be equally distributed into the two daughter cells. Segregation errors have severe consequences and lead to either cell death or the generation of aneuploid cells and may cause the formation of tumors or tumor promoting mutations in somatic cells. In meiosis, they provoke the generation of aneuploid embryos and/or spontaneous abortions. Trisomies in humans, such as trisomy 21 , are due to the missegregation of one chromosome in the first meiotic division in the oocyte. This review deals with the molecular mechanisms regulating the two meiotic divisions required for the generation of female haploid germ cells. Here we focus mainly on spindle assembly, and cell cycle regulation especially during the first meiotic division in mouse oocytes (excellent reviews have been written on the peculiar aspects of cell cycle regulation in meiosis II, such as the (SF arrest). $\diamond$

\section{REMERCIEMENTS}

Les auteurs s'excusent de ne citer qu'un nombre limité de publications en raison de restrictions de place. Nous remercions Marie-Hélène Verlhac, Khaled Hached et Jérémie Szeftel pour leurs commentaires sur le manuscrit. K.W. remercie C. Jessus pour son soutien. L'équipe de K.W. est soutenue par l'Inserm (subvention Avenir et PNRRE), l'ARC (subvention 3883), et le CNRS. M. E.T. est financée par une bourse NIH R01 CA107342 allouée à Prasad. V. Jallepalli.

\section{RÉFÉRENCES}

1. Cahill DP, Lengauer $\mathrm{C}, \mathrm{Yu}$ J, et al. Mutations of mitotic checkpoint genes in human cancers. Nature $1998 ; 392: 300-3$.

2. Lengauer C, Kinzler KW, Vogelstein B. Genetic instabilities in human cancers. Nature 1998 ; $396: 643-9$.

3. Hassold T, Hunt P. To err (meiotically) is human : the genesis of human aneuploidy. Nat Rev Genet $2001 ; 2: 280-91$.

4. Brunet $S$, Maro B. Cytoskeleton and cell cycle control during meiotic maturation of the mouse oocyte : integrating time and space. Reproduction (Cambridge, England) 2005; 130:801-11.

5. Verlhac MH, Lefebvre C, Terret ME, et al. L'ovocyte de souris et les particularités des divisions méiotiques. Med Sci (Paris) 2001 ; 17 : 1046-52.

6. Pellestor F. Maternal age and chromosomal abnormalities in human oocytes. Med Sci (Paris) $2004 ; 20: 691-6$.

7. Hodges CA, Revenkova $\varepsilon$, Jessberger R, et al. SMClbeta-deficient female mice provide evidence that cohesins are a missing link in age-related nondisjunction. Nat Genet 2005 ; $37: 1351-5$.

8. Kirschner M, Mitchison T. Beyond self-assembly : from microtubules to morphogenesis. Cell $1986 ; 45: 329-42$.

9. Ciciarello M, Lavia P. New CRIME plots. Ran and transport factors regulate mitosis. $E M B O$ Rep $2005 ; 6: 714-6$.

10. Eichenlaub-Ritter U, Vogt $\varepsilon$, Yin H, Gosden R. Spindles, mitochondria and redox potential in ageing oocytes. Reprod Biomed online $2004 ; 8: 45-58$.

11. Schuh M, Ellenberg J. Self-organization of MTOCs replaces centrosome function during acentrosomal spindle assembly in live mouse oocytes. Cell $2007 ; 130: 484-98$.

12. Brunet $S$, Maria AS, Guillaud $P$, et al. Kinetochore fibers are not involved in the formation of the first meiotic spindle in mouse oocytes, but control the exit from the first meiotic $M$ phase. J Cell Biol 1999; $146: 1-12$.
13. Dumont J, Petri S, Pellegrin F, et al. A centriole- and RanGTP-independent spindle assembly pathway in meiosis I of vertebrate oocytes. J Cell Biol $2007 ; 176: 295-305$.

14. Lefebvre C, Terret ME, Djiane A, et al. Meiotic spindle stability depends on MAPK-interacting and spindle-stabilizing protein (MISS), a new MAPK substrate. J Cell Biol 2002 ; 157 : 603-13.

15. Terret ME, Lefebvre C, Djiane A, et al. DOCIR : a MAP kinase substrate that control microtubule organization of metaphase II mouse oocytes. Development $2003 ; 130: 5169-77$.

16. Petronczki M, Siomos MF, Nasmyth K. Un menage a quatre : the molecular biology of chromosome segregation in meiosis. Cell 2003; 112: 423-40.

17. Gruber $\mathrm{S}$, Haering $\mathrm{CH}$, Nasmyth K. Chromosomal cohesin forms a ring. Cell $2003 ; 112: 765-77$.

18. Watanabe $Y$. Shugoshin : guardian spirit at the centromere. Curr Op Cell Biol 2005 ; $17: 590-5$.

19. Acquaviva C, Pines J. The anaphase-promoting complex/cyclosome: APC/ C. J Cell Sci 2006 ; 119: 2401-4.

20. Wassmann K, Benezra R. Mitotic checkpoints : from yeast to cancer. Curr Opin Genet Dev $2001 ; 11: 83-90$.

21. Irniger $S$. Preventing fatal destruction : inhibitors of the anaphasepromoting complex in meiosis. Cell cycle $2006 ; 5: 405-15$.

22. LeMaire-Adkins R, Radke K, Hunt PA. Lack of checkpoint control at the metaphase/anaphase transition : a mechanism of meiotic nondisjunction in mammalian females. J Cell Biol 1997 ; 139 : 1611-9.

23. Terret ME, Wassmann K, Waizenegger I, et al. The Meiosis I-to-Meiosis II Transition in Mouse 0ocytes Requires Separase Activity. Curr Biol 2003; 13: 1797-802.

24. Herbert $M$, Levasseur $M$, Homer $H$, et al. Homologue disjunction in mouse oocytes requires proteolysis of securin and cyclin Bl. Nat Cell Biol 2003; $5: 1023-5$.

25. Ledan $\varepsilon$, Polanski Z, Terret ME, Maro B. Meiotic maturation of the mouse oocyte requires an equilibrium between cyclin $B$ synthesis and degradation. Dev Biol $2001 ; 232: 400-13$.

26. Wassmann K, Niault T, Maro B. Metaphase I Arrest upon Activation of the Mad2-Dependent Spindle Checkpoint in Mouse Oocytes. Curr Biol 2003; 13: 1596-608.

27. Homer HA, McDougall A, Levasseur M, et al. Mad2 prevents aneuploidy and premature proteolysis of cyclin B and securin during meiosis I in mouse oocytes. Genes Dev $2005 ; 19: 202-7$.

28. Niault $T$, Hached $K$, Sotillo $R$, et al. Changing mad2 levels affects chromosome segregation and spindle assembly checkpoint control in female mouse meiosis I. PLoS One 2007 ; 2 : ell65.

29. Tsurumi C, Hoffmann S, Geley $S$, et al. The spindle assembly checkpoint is not essential for CSF arrest of mouse oocytes. J Cell Biol 2004 ; 167 : 1037-50.

30. Kudo NR, Wassmann K, Anger M, et al. Resolution of chiasmata in oocytes requires separase-mediated proteolysis. Cell 2006 ; 126 : 135-46.

31. Gorr IH, Reis A, Boos D, et al. Essential CDKl-inhibitory role for separase during meiosis I in vertebrate oocytes. Nat Cell Biol 2006 ; 8 : 1035-7.

32. Thornton BR, Toczyski DP. Precise destruction : an emerging picture of the APC. Genes Dev $2006 ; 20$ : 3069-78.

33. Reis A, Chang HY, Levasseur M, Jones KT. APCcdhl activity in mouse oocytes prevents entry into the first meiotic division. Nat Cell Biol 2006 ; $8: 539-40$.

34. Deng M, Suraneni P, Schultz RM, Li R. The Ran GTPase mediates chromatin signaling to control cortical polarity during polar body extrusion in mouse oocytes. Dev Cell 2007 ; 12 : 301-8.

\section{TIRÉS À PART}

K. Wassmann 


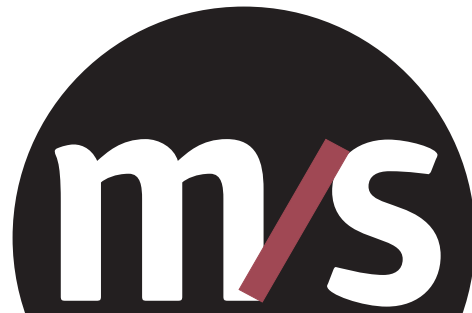

MEDECINE SCIENCES

Chaque mois, avec les articles de référence de $M / S$

\section{Chaque jour,} sur www.medecinesciences.org
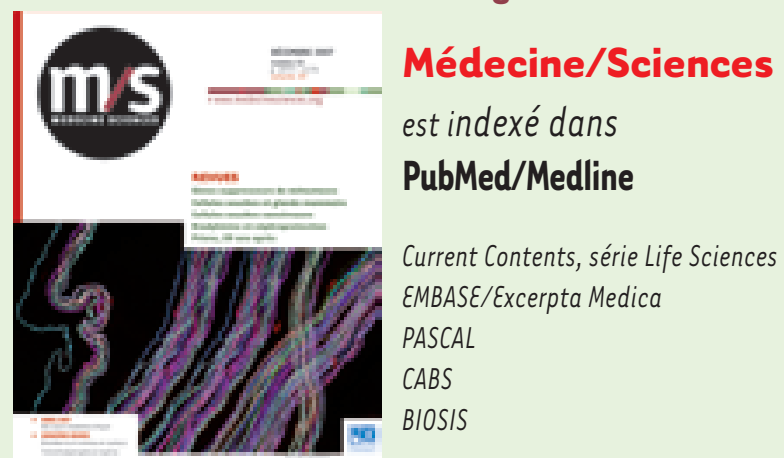

\section{PubMed/Medline}

Current Contents, série Life Sciences EMBASE/Excerpta Medica PASCAL

CABS

BIOSIS

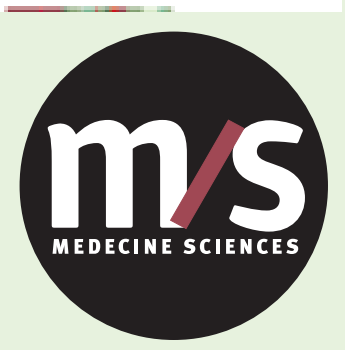

\section{$>$ Grâce à $m / s$, vivez en direct les progrès des sciences biologiques et médicales}

> Des articles rédigés par des médecins et des chercheurs reconnus sur la scène internationale qui posent avec rigueur les bases des débats scientifiques.

> Des synthèses, éditoriaux, dossiers techniques et analyses toujours replacés dans leur contexte pour que l'information soit la plus exacte, intelligible et objective.

> La dimension humaine privilégiée, avec l'analyse des retombées diagnostiques, thérapeutiques, la prévention et l'éthique liées aux nouvelles avancées.

> Un panorama clair et concis de l'actualité scientifique : des nouvelles, des brèves, des données chiffrées, des repères et perspectives pour qu'aucun fait significatif ne vous échappe.

Tarifs d'abonnement M/S - 2008

Mensuel - 10 numéros/an

Abonnez-vous

\section{à Médecine/Sciences}

Je souhaite m'abonner à $M / S$ :

Nom :

Prénom:

Adresse :

Code postal

Ville :

Pays :

E-mail-obligatoire :

Je choisis l'abonnement:

France

$U \varepsilon+$ autres

*Joindre un justificatif

$$
\text { Papier }
$$

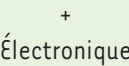

Particuliers

Électronique Papier

seul

$\square 170 €$

$\square 214 €$ $\square 118 €$

$\square 118 €$ $\square 160 €$

$\square 204 €$

\section{Mon règlement :}

$\square$ Par mail edk@edk.fr

Uniquement pour les paiements par carte bancaire

$\square$ Par fax en envoyant ce bulletin au 0155641394 Uniquement pour les paiements par carte bancaire

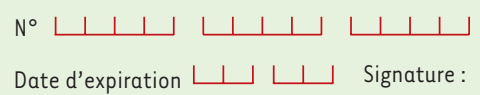

$N^{\circ}$ de contrôle au dos de la carte $\downarrow \downarrow$

$\square$ Par chèque à l'ordre de Médecine/Sciences, en envoyant ce bulletin à :

\section{Éditions EDK}

2, rue Troyon

92316 Sèvres Cedex, France

Pour recevoir une facture, cochez cette case $\square$ 